\title{
Tangence
}

\section{D'une mémoire à l'autre. Lecture du roman Le double conte de l'exil de Mona Latif-Ghattas}

\section{Louise Gauthier}

Numéro 59, janvier 1999

Écrivains d'ailleurs

URI : https://id.erudit.org/iderudit/025991ar

DOI : https://doi.org/10.7202/025991ar

Aller au sommaire du numéro

Éditeur(s)

Tangence

ISSN

0226-9554 (imprimé)

1710-0305 (numérique)

Découvrir la revue

Citer cet article

Gauthier, L. (1999). D'une mémoire à l'autre. Lecture du roman Le double conte de l'exil de Mona Latif-Ghattas. Tangence, (59), 49-61.

https://doi.org/10.7202/025991ar d'utilisation que vous pouvez consulter en ligne.

https://apropos.erudit.org/fr/usagers/politique-dutilisation/ 


\section{D'une mémoire à l'autre. Lecture du roman Le double conte de l'exil de Mona Latif-Ghattas}

Louise Gauthier

\section{La double appartenance}

Partir: geste irréversible et irrémédiable pour des millions d'êtres humains aujourd'hui déplacés hors de leur terre natale, tantôt librement, tantôt par la force des circonstances, et qui forment une masse de plus en plus imposante d'immigrés, d'exilés ou de réfugiés, porteurs pour toujours de la mémoire des origines en terre d'accueil. Ce mouvement de traversée des frontières, particulièrement important à l'heure actuelle à l'échelle mondiale, imprime aux pays d'accueil un visage de plus en plus métissé.

Le Québec n'échappe pas à cette transformation. Si l'immigration a de tout temps marqué son évolution, les nouveaux arrivants de pays autres qu'européens donnent aujourd'hui au visage culturel québécois des traits de plus en plus diversifiés. Le tissu social, d'assez homogène qu'il était jusqu'aux années soixante, est devenu de plus en plus hétérogène. Tous les secteurs de la vie culturelle témoignent de cette diversité, notamment la littérature. En effet, même si des écrivains nés ailleurs ont de tout temps pris la plume au Québec, l'explosion de ce phénomène est particulièrement remarquable depuis les années quatre-vingt ${ }^{1}$. Il se manifeste non seulement par une multiplicité d'œuvres produites par des auteurs de toutes les souches et de langues maternelles diverses, mais aussi par une prise de conscience, de la part de la critique, de la pluralité culturelle de la littérature du Québec.

Aborder la littérature actuelle signifie donc adopter l'angle d'une lecture interculturelle; oublier un instant le mythe de la pureté des origines pour se rendre disponible à la pluralité des cultures dans le texte. La démarche implique un changement de direction du regard: l'Autre n'est plus objet d'étude, mais sujet, porteur de parole. Se rendre disponible au regard de l'Autre

1 Pour plus de précisions sur ce sujet, voir Louise Gauthier, La mémoire sans frontières. Émile Ollivier, Naïm Kattan et les écrivains migrants au Québec, Sainte-Foy, IQRC, coll. "Culture et société", 1997, 143 p. 
50

amène la découverte du pays habité par l'imaginaire et tracé aux contours des différentes mémoires qui s'y rencontrent.

Les textes des auteurs d'ici issus d'une autre contrée, d'une autre culture et souvent d'une autre langue, sont très révélateurs des traces laissées par la perte du pays natal et par le passage d'une culture d'origine à la culture d'accueil. L'exil apparaît comme la source d'inspiration de ces écrivains, avec une intensité variable selon le degré d'adaptation à leur nouvelle identité. Émile Ollivier et Naïm Kattan, par exemple, après trente ans au Québec, font encore de l'exil leur inspiration, mais avec un recul influencé par l'intégration à un nouveau terreau, à une nouvelle société, bref à une nouvelle culture. Chez ces auteurs, la mémoire d'origine, de longue durée, revit en force, intimement liée à la mémoire nouvellement acquise. Chacun affirme porter en lui une double identité et vivre dans la division de l'être, cherchant à se définir sous le signe du passage. Pierre Monette fait remarquer à juste titre que "[1]'identité immigrante se trouve moins dans l'appartenance que dans une partance où le plus urgent devient de prendre pied, de prendre place, de prendre racine au lieu de s'en chercher " ${ }^{2}$. Mais, même transplanté de longue date et écrivant au présent, l'immigrant continue de se développer à partir d'un système radiculaire d'origine intégré à un nouveau terreau; s'il y développe de nouvelles racines, le thème de la mémoire est néanmoins omniprésent chez lui. Pierre Nepveu parle à juste titre de "malaise au pluriel" qu'il explicite ainsi: "malaise de l'appartenance, de l'arrachement, de la distance, de l'exil et, surtout, malaise de la mémoire et de l'impossible oubli " ${ }^{3}$. Si Émile Ollivier observe en lui-même deux tonalités qui cheminent parfois côte à côte, et parfois s'enchevêtrent, au point où il se demande s'il n'est pas "un schizophrène heureux" ", Naïm Kattan affirme de son côté que choisir l'exil, c'est "opter lucidement [...] pour la division de l'être" 5 . On pourrait multiplier ainsi les exemples de la double appartenance.

2 Pierre Monette, L'immigrant Montréal, Montréal, Triptyque, 1994, p. 43

3 Pierre Nepveu, "L'impossible oubli. Le vrai défi du pluralisme est celui non pas d'une négation mais d'une relativisation de la mémoire", Le Devoir, 10 juin 1997, p. A9.

4 Émile Ollivier, "Du bon usage de l'exil et de la schizophrénie", Le Devoir, 5 novembre 1983 , p. xiv.

5 Naïm Kattan, Le réel et le théâtral: essai, Montréal, HMH, 1970, p. 172. 


\section{Le double conte}

Une auteure a su l'exprimer de façon particulièrement émouvante: Mona Latif-Ghattas, qui a quitté l'Égypte à dix-neuf ans, peut aujourd'hui affirmer: "Je suis Orient et Occident. [...] Je suis double. J'ai une double destinée et une double vie. Je les ai totalement assumées. [...] Il n'y a plus de combat [...] entre les cultures, entre ces mémoires [...]." 6 Plonger dans son univers romanesque et poétique permet de côtoyer de façon privilégiée l'expérience transculturelle. Le texte est témoin d'une existence fractionnée, du passage, de l'entre-deux. Parmi l'ensemble de son œuvre déjà abondante et riche, nous nous intéressons particulièrement, dans le présent article, au roman intitulé Le double conte de l'exil où, sur une double trame formée des récits de Fêve et de Madeleine, nous pouvons suivre la rencontre de deux êtres issus de deux mondes étrangers qui tisseront entre eux des liens "têtus", "lianes entremêlées dans la chaleur de la mémoire sur lesquels se reposent les oiseaux migrateurs" (p. 10). Le récit principal met en scène Madeleine, montréalaise d'origine amérindienne, à jamais marquée par sa rencontre avec Fêve, réfugié du désert d'Anatolie, qui écrit par bribes les souvenirs qui remontent à sa mémoire. Les deux récits s'entrecroisent: l'un sous une forme narrative classique, l'autre sous la forme d'un conte oriental fantastique. Ainsi se côtoient et s'interpénètrent deux mémoires culturelles issues de l'Occident et de l'Orient. Ainsi s'apprivoisent l' aïeule d'un continent de neige" et "ce descendant rebelle fuyant le feu doux d'un désert d'Anatolie" (p. 10), l'Ici et l'Ailleurs. Déjà le prologue contient l'essence de ce qui va suivre.

Madeleine, autrefois Manitakawa, vit dans l'anonymat et le silence à Montréal, durcie et secrète depuis le jour où, à l'âge de douze ans, elle s'est fait violer à l'arrière de la taverne tenue par sa mère. Robuste, elle exerce le dur métier de buandière, travail habituellement réservé aux hommes. Elle vit ainsi jusqu'au jour où, le long des quais, elle découvre un jeune homme trempé, rescapé sans doute de la cale d'un bateau, qu'elle ramène chez elle. C'est là que Fêve se remet peu à peu du choc de l'exil et commence à saisir les mots et les coutumes que Madeleine lui enseigne patiemment; il appréhende peu à peu son nouvel univers

6 Monique Grégoire, "Mona Latif-Ghattas. De l'exil à l'appartenance", Nuit blancbe, $\mathrm{n}^{\circ} 55$, mars-avril-mai 1994, p. 32.

7 Mona Latif Ghattas, Le double conte de l'exil, Montréal, Boréal, 1990, 171 p. 
52

au point de se trouver un emploi et de faire une demande d'asile politique. Cependant, malgré les efforts de Madeleine devant une "erreur inhumaine" ( $p, 158$ ), la demande est refusée et Fêve est expulsé. Bouleversée par cette injustice, Madeleine sent remonter en elle les mots méconnus des langues amérindiennes et retourne à la réserve où elle sera intégrée au Conseil des anciens.

La dualité inscrite au cour de l'œuvre se manifeste tout d'abord dans la structure même du roman. La narration principale, à la troisième personne, raconte le présent du récit: la relation entre Fêve et Madeleine, leur rapport à la vie et aux gens de Montréal, la situation des immigrants. Dans la première trame vient s'insérer le journal de Fêve, à la première personne et en italique, véritable conte arabe où un fabuleux conteur, mû par la douleur de l'insupportable, permet à l'homme blessé d'émerger de la mémoire et de se libérer "dans le tracé écorché de son passage noyé par la fuite des jours" (p. 28). L'œuvre allie donc culture orale et culture écrite, culture d'origine et culture d'accueil, mémoire de longue durée et mémoire plus actuelle, Orient et Occident. Elle est fondée en outre sur un double regard, celui du natif sur l'étranger et celui de l'exilé sur "l'espace où le destin [l]'a réfugié" (p. 27).

Les thèmes de l'exil et du passage sont privilégiés chez Mona Latif-Ghattas, comme chez Émile Ollivier, Naïm Kattan et la plupart des écrivains migrants. Il arrive que le titre même atteste ces préoccupations, comme celui du roman d'Ollivier, Passages, ou de deux ouvrages de Latif-Ghattas: Quarante voiles pour un exil ${ }^{8}$ et Le double conte de l'exil. Le récit de Fêve s'ouvre sur la douleur vive qui s'exprime en laissant surgir l'homme "qui perce [sa] mémoire pour revivre dans l'exil" (p. 27). Tandis que remontent les souvenirs du désert, il s'applique à apprendre le nouveau pays; le désarroi fait place à une adaptation graduelle; il apprend à aimer les fèves au lard, à dresser la table selon les instructions de Madeleine, à se "calfeutrer avant de sortir" (p. 89) pour faire face au vent, en attendant de pouvoir se payer le manteau doublé de mouton dont il rêve. Bien sûr, il travaille au noir, dans les égouts par surcroît, mais espère qu'un jour, bien habillé, "il aurait l'air de tout le monde" (p. 90). Il découvre les téléromans, la neige, l'argent, arrive à faire les courses, la cuisine; il lie connais-

8 Quarante voiles pour un exil: récits et fragments poétiques, Laval, Éditions Trois, coll. "Topaze ", 1986, 105 p. 
sance avec d'autres voyageurs de l'autobus "venus des quatre coins du monde" (p. 135). Après six mois, il parle correctement le français, regarde des films et écoute des concerts, visite les régions du Québec avec Madeleine. Tout au long de son apprentissage, il poursuit sa démarche d'écriture, poussé "par un instinct de survie" qui l'incite à "ancrer son image" pour arriver enfin "à exister"; "le présent ne lui accordera ses lettres de créance et un certain repos du coeur qu'au moment où il lui aura offert en échange la trame de son passé aussi halluciné qu'il puisse paraître". Et Madeleine à ses côtés fait figure de "passerelle magique vers le présent" (p. 92). Mais le rêve ne pourra se concrétiser puisque sa situation "d'illégal" jouera contre lui qui se refuse aux manigances utilisées par d'autres pour contourner la loi. La vérité et l'honnêteté se sont avérées des pièges pour Madeleine et Fêve, trop naïfs pour le système. Ni le bombardement de sa maison, ni les humiliations, ni le vol de sa terre, ni le viol de son amour, ni sa recherche du rêve perdu ne sauront convaincre l'agent d'immigration de la vérité du récit de Fêve: "une vérité si cruelle qu'elle avait l'allure d'un conte" (p. 139). Le séjour de Fêve à Montréal aura été lui-même un passage entre l'aller et le retour.

Un double système d'images et de références animent le texte du roman. Le récit de Madeleine traduit bien l'atmosphère de Montréal en novembre, le froid humide, le vent, la pluie, les premiers brins de neige opposés à la chaleur de l'autobus ou de la buanderie. La soupe aux pois verts ou le rôti de porc, les fèves brunes dans la mélasse et les cubes de lard, accompagnés de Coca-Cola, suggèrent des odeurs rassurantes pour un Fêve effrayé et dépaysé. Au cours du récit, les saisons passent avec leurs couleurs et leurs odeurs. Le monde enterré sous la neige fait place au printemps, à la lumière du jour qui se prolonge tandis que le pommier en fleurs embaume. Fêve a vécu jusque-là dans le noir, au propre comme au figuré: travailleur au noir, il part aux petites heures du matin, descend dans un trou pour travailler sous terre, dans les égouts, et n'en ressort que le soir lorsqu'il fait noir. Ce n'est qu'au printemps qu'il découvre la lumière en même temps que l'espoir d'être reçu comme immigrant. Mais la chaleur accablante de juillet étouffe l'espoir et le ramène au désert.

Conte arabe où les images foisonnent, le récit de Fêve alterne avec celui de Madeleine, surgi des profondeurs de la mémoire, il évolue au rythme des réminiscences, par bribes, sur une toile porteuse des images de l'Orient. Le récit est mis dans la bouche d'un 
54

conteur qui parle à la place de Fêve; ou plutôt de la douleur qui "perce [sa] mémoire" pour faire surgir l'homme du lointain, "conteur inguérissable, qui invente tout, qui n'invente rien, qui transporte la beauté la plus vive et la plus atroce laideur d'un bout à l'autre de ses errances" (p. 27) pour faire émerger "dans la pitié de [son] coeur" cet homme "dans son hallucinante vérité. Le libérer innocemment dans la violente innocence d'une naissance, le laisser re-commencer comme si jamais encore il n'avait eu lieu" (p. 28). Fêve prend le risque de se détourner du présent et de se perdre dans les méandres du souvenir. Le jeune homme se dédouble pour être à la fois le conteur et le personnage raconté; il cerne alors peu à peu le jeune homme qui se présente sous un soleil de plomb dans les sables du désert, son univers, là où "le flot du mot crève son coeur pour jaillir dans le désert" (p. 29). Les images opposeront dès lors l'eau au sable, la mer au désert, l'oasis à la sécheresse; pour conter "l'innommable" qui le poussa du désert à la mer, lui pour qui le sable du désert est devenu sable de grève; pour confronter ou entremêler les éléments clés de son "passage noyé" (p. 28) vers une nouvelle destinée potentielle.

Une importance capitale est accordée à une couleur, le vert, omniprésent: le vert des figuiers, celui du désert et de la plage, avec une insistance sur le "vert désertique, retenez la couleur, retenez-la", celle qu'on ne retrouve que sur les figuiers et nulle part ailleurs, celle "des cendres du souvenir d'un fruit " (p. 40); car c'est aussi celle des yeux de Mariam Nour, "quand elle parlait", et celle de la mer, si présente. Mais aussi celle dont étaient vêtus ces hommes, venus des vallées fertiles camper dans le désert, qui se sont jetés sur Mariam Nour; une goutte de rubis mêlée alors au sable formera un caillou rose, seule couleur du désert blanc de sable beige. Tout aussi important est le ton de vert, voilé de gris pour les figuiers; le ton est primordial tant dans la nature que dans la vie et dans le ton du récit: "il y a un ton de vert comme il y a un ton de voix pour aimer et pour blesser. La vie se tisse et se défait selon le ton des choses. [...] Pourvu que ma mémoire voyageuse se maintienne dans la bonne tonalité" ( $p$. 41). Le vert qui teinte la mémoire de Fêve fait toute la différence comme "le vert des déserts reste une énigme à celui des forêts" (p. 42). Le vert qui symbolise toute l'étrangeté par une simple nuance, comme la couleur et le ton distinguent les hommes.

Si la couleur est un outil de premier ordre utilisé par l'auteure pour rendre perceptible l'atmosphère du désert d'origine, l'image 
de la figue l'est tout autant. Fruit du désert, elle vient concrétiser ou symboliser la douceur perdue. D'ailleurs, la voix de Mariam Nour ne coulait-elle pas "comme le miel des figues" (p. 41)? Dans ce désert peuplé uniquement de figuiers, Mariam Nour deviendra "figue accrochée aux figuiers verts" ou encore "figue de soie sur la mémoire" (p. 96). Et un soir de froid, à Montréal, Fêve préparera pour Madeleine un repas de son pays de soleil, "l'agneau rôti des jours de fête", clôturé par un plat de quatre figues, achetées à prix d'or, en disant "que c'était le fruit le plus doux que la terre ait porté" (p. 124).

Ainsi les références des deux récits en alternance font passer le lecteur d'un univers à l'autre, d'une mémoire à l'autre. Le contraste entre le pays chaud désertique et le pays froid de neige se fait sentir tout d'abord par les images, les couleurs et les sensations de chaleur et de froidure associées au climat. La nourriture et les odeurs ont aussi une forte connotation identitaire, le rituel des repas étant une occasion de prédilection pour créer des liens et apprivoiser le quotidien de l'autre. Le double conte fait alterner le présent et le passé : présent québécois et passé oriental, double mémoire qui peu à peu s'entremêlent et permettent à Madeleine de laisser surgir à son tour des images d'une mémoire originelle enfouie au fond d'elle-même. L'issue tragique de l'histoire sépare les deux êtres et scelle le double retour aux origines. Madeleine n'aura de cesse, depuis ce jour, de raconter aux enfants les contes et histoires vraies, d'Indiens ou de désert, "des contes, beaux et cruels, des multicontes de l'exil" (p. 168).

\section{La double langue}

Toutes les caractéristiques mentionnées plus haut sont imprimées dans la langue qui supporte le roman. À cet égard, quelques réflexions méritent une parenthèse. Tout d'abord, beaucoup d'écrivains migrants du Québec, comme Mona Latif-Ghattas, viennent non seulement "d'une autre culture [mais] vraisemblablement d'une autre langue vernaculaire, même si la socialisation scolaire s'est faite en anglais ou en français" " Plusieurs ont choisi d'écrire en français, faisant de cette langue leur patrie car, nous rappellent-ils, pour un écrivain la véritable patrie c'est la langue.

9 Régine Robin, "Langue-délire et langue-délit", Discours social/Social Discourse, vol. V, nos 3-4, été-automne 1993, p. 24. 
Mais la mémoire d'origine survit toujours en eux et se manifeste dans la langue d'écriture. Ainsi, le français utilisé par l'écrivain porte l'empreinte d'un apport culturel différent, dans l'esprit, le tythme ou le vocabulaire. L'écrivain adopte une langue avec ses règles et sa mémoire, et y apporte la marque d'une autre mémoire culturelle. On se retrouve donc devant un plurilinguisme plus ou moins accentué, une hétérogénéité de la langue qu'ont déjà étudiée Régine Robin et Lise Gauvin ${ }^{10}$. La langue française s'enrichit d'un apport extérieur. Il y a échange des codes.

Le double conte de l'exil en est une démonstration éloquente. La construction elle-même, en deux récits, permet la rencontre de deux mondes: l'Occident et l'Orient, plus précisément deux lieux, Montréal et le désert d'Anatolie. La langue d'écriture est le français dans les deux cas, mais dans des registres très éloignés l'un de l'autre. Le présent du roman, raconté par le narrateur externe, suit tout à fait le génie du français classique, ses procédés. En lisant l'histoire de Madeleine et de Fêve le Fou à Montréal, le lecteur québécois est tout à fait familier avec la langue utilisée. Il en va tout autrement pour le récit de Fêve qui recèle une atmosphère résolument exotique. Non seulement par les images que nous avons évoquées pécédemment, ou par la langue généreusement fleurie, mais par le "ton" lui-même utilisé par le conteur. Car il s'agit bien là d'un conteur comme il s'en trouve dans la plus pure tradition arabe; et, bien au-delà de cette culture, comme il en existe dans toutes les traditions orales, qu'elles soient antillaise ou africaine. Il ne s'agit donc pas seulement de parsemer le récit d'un vocabulaire particulier ou d'images exotiques pour marquer les différences de langues. C'est l'essence même de la langue qui est différente. Édouard Glissant, parlant de la créolisation, exprimait ces idées qui peuvent s'appliquer à notre propos: "nous sommes des praticiens de l'oralité. [...] Le conteur antillais s'appelle un maître de la parole. [...] Le conteur créole utilise des procédés qui ne sont pas dans le génie de la

10 Lise Gauvin, "La surconscience linguistique de l'écrivain francophone: position des revues québécoises ", Revue de l'Institut de sociologie, 1990-1991, p. 83-101 ; "Une surconscience opérante: les stratégies textuelles des années 80 ", Discours social/Social Discourse: La langue fétiche, vol. $5, \mathrm{n}^{\mathrm{OS}} 3-4$, été-automne 1993, p. 139-157 ; "Poétiques de la langue et stratégies culturelles" dans Claude Duchet et Stéphane Vachon [dir.], La recherche littéraire. Objets et métbodes, Montréal/Paris, XYZ éditeur/Presses universitaires de Vincennes, 1993, p. 333-341. 
langue française, qui vont même à l'opposé: les procédés de répétition, de redoublement, de ressassement, de mise en haleine " ${ }^{11}$. La littérature francophone internationale nous fournit de multiples exemples de l'oralité imprégnant l'écriture. L'auteur le plus connu est sans doute Tahar Ben Jelloun qui peut construire un roman sous la forme d'un long récit au cours duquel le conteur s'adresse à la foule ou est interrompu par ses auditeurs, contredit même par eux ${ }^{12}$.

Dans Le double conte de l'exil, Fêve se présente comme "un conteur fabuleux, [...] sans lois préfigurées" (p. 29). Il annonce dès le début la forme de son récit, prévenant le lecteur de l'étrangeté liée au rituel du conte:

Cet homme est un Conteur c'est-à-dire un homme qui a peur de mourir avant d'avoir conté. [...] S'il arrive à éclaircir sa voix, il soufflera des images colorées. Ses cordes vocales acrobatiques insuffleront alors la mouvance aux images et le conte vivra, dans sa chronologie anarchique, sa ponctuation déréglée et surtout, surtout, l'espace de ses silences hâbleurs, là où ne cessent de frémir de genèse en genèse les vérités humaines, à travers le surprenant écho de l'invraisemblance. (p. 30)

Le conteur adopte la démarche circulaire du conte, revenant souvent au point précédent pour avancer encore, éclaircir les faits encore un peu plus, répéter, rappeler, parsemant le récit de "Je disais donc..."; cherchant toujours le juste ton: "Je parle à mivoix pour ne pas effaroucher Mariam Nour [...]. Tant qu'il n'en tient qu'à moi, je raconterai à mi-voix" (p. 40, 41). Il s'adresse à son auditoire fictif, tantôt pour capter ou maintenir son attention: "Retenez la couleur"; "écoutez, écoutez"; tantôt pour le prier de ne pas l'interrompre: "Et si quelqu'un a l'intention d'interrompre le conte je vais perdre la voix" (p. 119). Car le conte est une forme interactive qui peut se construire à partir des rumeurs ou des interventions de l'assistance; d'autres conteurs peuvent prendre le relais pour contredire, commenter ou poursuivre l'histoire. Tous ces éléments se retrouvent dans le journal de Fêve: «il y a des vieilles langues, dans le village désertique, qui racontent que [...]. D'autres vieillent langues racontent que [...]. (p. 109). Et

11 Lise Gauvin, "L'imaginaire des langues. Entretien avec Édouard Glissant", Études françaises, vol. XXVIII, $\mathrm{n}^{\text {os }} 2-3$, automne-hiver 1992-1993, p. 17, 18.

12 Voir en particulier L'enfant de sable de Tahar Ben Jelloun, Paris, Seuil, coll. "Points", n R296, 1988, c1985, 208 p. 
plus loin: "Alors, laissez-moi dire encore [...]" (p. 129). Puis finalement :

Il y a quelqu'un qui reprend mon conte.

Je vais bientôt perdre la voix.

Ainsi se dévide l'écheveau des contes désertiques.

Mais je vais encore conter tant que mon souffle dure.

Tant que dure ma voix.

Quand la voix de Fêve s'éteint, c'est Madeleine qui prend le relais. D'abord pour raconter à un enfant le sort réservé aux Indiens d'Amérique. Et plus tard, longtemps après la déportation du réfugié, pour raconter aux enfants de la réserve, autour du feu de bois, "des contes, beaux et cruels, des multicontes de l'exil" (p. 168) s'inspirant des écrits de Fêve conservés dans un sac de plastique vert. Ainsi se transmet la parole; ainsi s'échangent les codes. Et comme les contes peuvent suivre plusieurs déroulements, celui-ci se conclut sur plusieurs pistes: "Choisissez... C'est ainsi que finissent les contes désertiques (p. 171)."

C'est de cette façon que Mona Latif-Ghattas intègre les langues de l'oralité et de l'écriture, les images de l'Orient et de l'Occident. Ces langues au début se côtoient pour finalement s'interpénétrer. L'un apprivoise peu à peu les mots de la réalité québécoise, l'autre respire au rythme de la forme du conte. Il y a échange dans la langue comme dans les autres aspects de la vie; chaque système s'enrichit au contact de l'autre.

\section{La multiplicité des regards}

D'ailleurs le contact avec l'Autre est au cour du roman et mérite qu'on s'y attarde. Car il n'y a pas que Madeleine et Fêve le Fou qui y évoluent. Plusieurs personnages gravitent autour d'eux, chacun représentant un certain niveau d'étrangeté par rapport aux autres, pour former un tissu social disparate où se manifeste une multiplicité de regards. Mona Latif-Ghattas compose ici un jeu de directions de regards, chacun jaugeant et jugeant son voisin, comme pour démontrer par l'exemple qu'on est toujours l'autre de quelqu'un. Il y a des degrés dans l'étrangeté comme dans toute chose. Fêve est à même de le constater, lui qui a été congédié de son travail d'éboueur lors du passage d'inspecteurs chargés de dépister les travailleurs au noir; il ne sera pas réembauché, entre autres choses parce que "d'autres réfugiés plus réfugiés que lui étaient prêts à travailler pour un moindre salaire» (p. 89). 
Le petit monde qui évolue dans le roman est à l'image du Montréal de cette fin de siècle: coloré et de toute provenance. $\grave{A}$ la buanderie, les travailleurs et travailleuses semblent majoritairement des Québécois "de souche", Madeleine elle-même représentant la lignée la plus ancienne. Ils sont dépeints sous les traits des Trois Clara dont le regard décapant vise à la fois Madeleine, dont le teint est un peu trop rouge et qui a quelque chose d'étrange dans le regard, et le jeune Asiatique qui sent un peu trop "l'egg roll" (p. 54). En effet, "la ligue des Trois Clara était implantée dans la buanderie depuis quelques décennies"; Madeleine était arrivée bien avant elles, toute jeune encore, alors que sa peau était encore très rouge et ses cheveux, très noirs. Elle avait assisté à l'intégration des Trois Clara dont "les noms se suivaient sur la liste de paye la plus ancienne de l'établissement "; noms qui désignent eux-mêmes trois origines distinctes qu'on reconnaîtra sans peine: Légaré, Lindsay, Leibovitch. Ainsi, à elles quatre, ces femmes représentent l'historique du peuplement du pays. Mais les Trois Clara ont tôt fait de se regrouper pour représenter l'ensemble de ce qui peut exister comme sentiments xénophobes:

Fortes de leurs similitudes et de leur ancienneté désormais irréfutable, elles s'octroyaient le droit de dévisager tout nouveau venu, de le scruter, de commenter ses comportements, de pointer du doigt sa différence, d'épier ses misères, de salir sa beauté si elle les poussait dans l'ombre, d'amoindrir ses qualités quand elles menaçaient de mettre à jour leurs lacunes, enfin de bâtir sa réputation. (p. 54)

La couleur intervient encore ici car les Trois Clara se sont "regroupées avec le temps, presque naturellement, par affinité de couleur. Elles avaient toutes les trois les cheveux roux" (p. 54). Leur alliance cimentée par la ressemblance cristallise tout ce qui peut exister comme sentiment hostile à l'étranger, comme réaction instinctive de défense devant une vague menace née de l'inconnu. "De leur angle de vision elles perçoivent des choses qui les déboussolent et aiguisent en elles une sorte de haine indéfinie, une haine, comme on dirait, épidermique, épidémique, qui peut même devenir contagieuse" (p. 100). La crainte suscitée par l'impassibilité et l'impénétrabilité de leur jeune collègue vietnamien, alimentée par la peur de se faire déloger, s'amplifie et se transforme pour atteindre toutes les minorités aux comportements différents, qu'il s'agisse des Marocains, Sikhs, Haitiens, Égyptiens, Chiliens, Salvadoriens, Colombiens, Iraniens, "Juifs à boudin", Turcs, "et quoi encore, quoi encore, les urgences des hôpitaux. 
60

engorgées, le métro où on se croit dans la tour de Babel, mon Dieu, mon Dieu" (p. 103).

Madeleine ne comprend pas cette cabale. Elle se tient en marge, elle qui "n'a jamais fait partie d'aucune majorité" (p. 104), pour observer cette défense contre "le présumé envahisseur"; "ou alors, sans le savoir, elle refuse de voir ce qui pourrait ressusciter des blessures mortes". La buanderie est le thêâtre où évoluent les différentes mémoires, les plus récentes étant les plus rapides à surgir; mémoires qui allument les différents regards qui se croisent et palpitent selon les racines qui les nourrissent.

Pendant ce temps, Fêve apprend la vie de clandestin. Il côtoie d'autres hommes de la même condition, à commencer par Americo, le Salvadorien, qui sera expulsé avant lui. Le matin, "dans l'autobus gris", il se mêle à "une armée de travailleurs venus des quatre coins du monde. Du Sud de l'Amérique, du Nord de l'Afrique, d'Asie ou du Moyen-Orient" (p. 135) envers lesquels il éprouve à la fois des sentiments de fraternité et de méfiance. De leurs conversations il apprend toutes les combines illicites pour arriver à s'implanter au pays. Terrorisé à l'idée de rencontrer l'agent d'immigration, il ne sait plus quels mots trouver pour faire croire à sa vraie histoire. Par son regard se dessine l'image du pays d'accueil, la faiblesse du plus démuni face au pouvoir de la société en place dont il ne mâ1trise pas les mécanismes; la dichotomie entre les critères qui forcent un homme à s'abandonner à la mer comme seul espoir de survie et ceux des étrangers qui, dans leur chez-eux, jugent de son acceptabilité. Le seul ton des deux récits démontre l'impossibilité de joindre les deux univers malgré la bonne volonté du réfugié dont tous les efforts tendent vers un seul but: s'adapter.

\section{Au carrefour des mémoires}

Une ouvre comme celle que nous venons de parcourir confronte le lecteur à la rencontre de l'étranger, l'oblige à suivre des regards de provenances diverses. Le double conte de l'exil est un microcosme de la littérature du Québec porteuse des multiples mémoires individuelles qui la composent. Il invite également à dépasser l'individu pour se pencher sur le phénomène de la mémoire collective qui reçoit et canonise les ouvres en un corpus susceptible d'alimenter ensuite l'imaginaire des lecteurs, et, conséquemment, de donner au lectorat l'aptitude à recevoir et à décoder des textes porteurs de différents systèmes de référence. 
François Paré s'attarde sur la question de l'intégration des mémoires individuelles à la mémoire collective ${ }^{13}$. Il insiste sur l'importance des "pratiques remémorantes " qu'il partage en deux formes: tout d'abord, la littérature elle-même puisqu'elle permet "de disséminer dans toute communauté des signes de l'identité collective, de sorte que la mémorialisation des ouvres semble être, par surcroit, aux yeux du groupe lui-même une mémorialisation des symboles de la collectivité" (Paré, p. 43). Mais cette première mémorialisation n'est pas la principale pratique pour cimenter une collectivité nationale; "c'est plutôt l'acheminement des œuvres et des écrivains dans une histoire réappropriée [ou] histoire mémoriale" qui joue ce rôle. On pourrait parler, selon Paré, "de mémorialisation de la mémorialisation", processus selon lequel s'effectue la promotion d'auteurs et d'œuvres dans un discours décalé où on parle sur l'auteur, sur sa parole.

Ainsi se dessine la responsabilité de la critique, de l'enseignement et des autres pratiques sociales de l'institution littéraire qui introduisent les ouvres parmi les objets du savoir d'une collectivité ; qui lui accordent une place dans le discours social et contribuent à façonner une littétature nationale. La période actuelle se caractérise précisément par une redéfinition de l'héritage qui façonne la mémoire collective du Québec, par un questionnement sur l'essence et la notion de littérature nationale qui évolue au rythme de l'histoire et des différentes mémoires qui la constituent. La fréquentation d'écrivains comme Mona Latif-Ghattas ne fait qu'aviver cette sensibilité à la complexité mouvante d'un tel héritage.

13 On pourra se référer à ce sujet aux Littératures de l'exiguité (Hearst, Le Nordir, coll. "Essai Le Nordir", 1992, 175 p.). 